\title{
Multi-Tone EMC Testing Strategy for RF-Devices
}

\author{
Alessandro Biondi ${ }^{\# 1}$, Hendrik Rogier ${ }^{\# 2}$, Dries Vande Ginste ${ }^{\# 3}$, Daniël De Zutter ${ }^{\# 4}$ \\ \# Department of Information Technology, Ghent University \\ Sint-Pietersnieuwstraat 41, B-9000 Gent, Belgium \\ ${ }^{1}$ Alessandro.Biondi@ugent.be; ${ }^{2}$ Hendrik. Rogier@uGent.be; \\ ${ }^{3}$ Dries.VandeGinste@UGent.be; ${ }^{4}$ Daniel. DeZutter@UGent.be
}

\begin{abstract}
In low-cost miniaturized electronic systems, filters are often omitted in front of active non-linear components, potentially resulting in unwanted intermodulation products in the band of operation. Current immunity tests most often use a single-frequency source and are hence not able to capture all relevant intermodulation products. Relying on an anechoic chamber as test facility and using multiple-tone excitation from a dual-source network analyzer, we present an advanced test methodology to evaluate in-the-band leakage of out-of-band undesired frequencies. To demonstrate our approach we use a frequency-selective active textile antenna with integrated nonlinear low-noise amplifier.
\end{abstract}

\section{INTRODUCTION}

The research presented in this paper was triggered by the increasing interest in the development of compact, low-cost and low-power microwave systems in which filters in front of active devices [1] are often omitted. A typical example is software defined radio, where a very broadband signal is received by the antenna and directly amplified before being converted to the digital domain, where further processing, including filtering, takes place [2]. Non-linearities, combined with the lack of shielding and filtering, may compromise performance [3] due to in-the-band leakage of out-of-band undesired signals interfering with the desired signal. This issue in particular arises when several sources illuminate devices under test [4]. Hence, in this contribution, we present an experimental strategy to model and test RF devices under multiple frequency sources illumination in the presence of out-of-band frequency components leaking into the band of interest. In Section II the different mechanisms that lead to in-the-band leakage of undesired signals via intermodulation (IM) distortion are discussed allowing to define suitable test strategies. Section III discusses the practical implementation of these strategies for a representative circuit, consisting of a textile antenna combined with a low-noise amplifier (LNA) that creates intermodulation distortion (IMD) due to its nonlinearity.

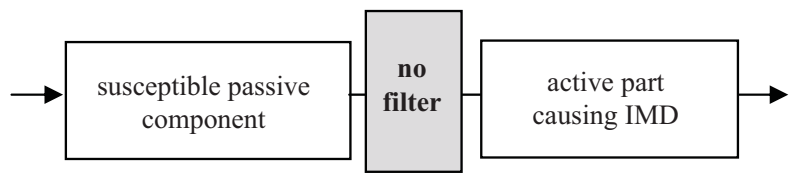

Fig. 1: Device Under Test (DUT)

\section{EVALUATION OF A DUT UNDER MULTIPLE SOURCE ILLUMINATION}

\section{A. Evaluation scenarios}

Fig. 1 shows a typical Device Under Test (DUT) susceptible to multi-frequency signals as it consists of a concatenation of a susceptible linear device and a non-linear component creating IM products. We suppose that no filter is present between the susceptible device and the non-linear component allowing undesired out-of-band signals to leak into the frequency band of operation. Figs. 2, 3 and 4 show the three illumination scenarios that generate in-the-band intermodulation distortion in the DUT due to multi-frequency signals. In Fig. 2 (scenario 1) a transmitter illuminates the DUT with a single frequency component or tone at $f_{0}$, and a higher-order (the second-order in the figure) harmonic falls into the band of interest $\left[f_{L}, f_{H}\right]$ as an unwanted signal. As the unwanted signal can no longer be removed by filtering it is necessary to test whether the power of this in-the-band disturbance remains small enough compared to the desired signal. Fig. 3 (scenario 2) considers illumination by two tones, a desired one $f_{1}$ falling in the band, and an unwanted one $f_{2}$ falling out-of-band, radiated by the co-located sources. In this second scenario the two frequency components result in an intermodulation product falling in the band of operation of the device. The power of the in-the-band interference must be monitored to validate the EMC behavior of the DUT. Fig. 4 shows the third scenario in which a high power out-of-band transmitter at frequency $f_{2}$ illuminates the DUT. Because of its high power, the LNA gets desensitized, i.e. gain and linearity decrease, resulting in a desired signal that is less amplified and hence more difficult to detect. Also this scenario should be accounted for in EMC aware design.

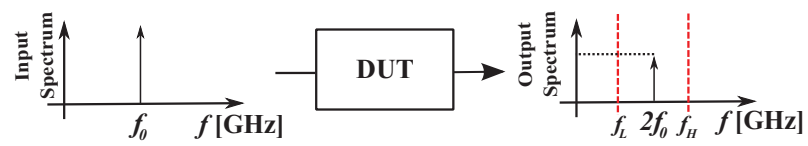

Fig. 2: Scenario 1: higher order harmonics in the DUT output spectrum.

\section{B. Test set-up and typical DUT example}

The three scenarios discussed above are now illustrated and put to the test by considering the example DUT depicted in Fig. 5, i.e. an active textile antenna [5]. In that case, the susceptible component is the passive antenna, and the 


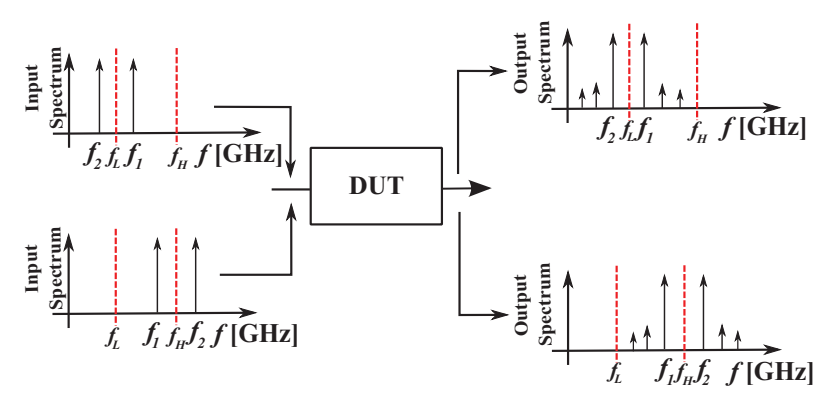

Fig. 3: Scenario 2: intermodulation products in the DUT output spectrum.

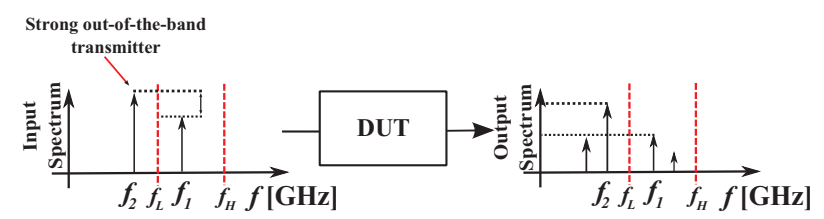

Fig. 4: Scenario 3: desensitization due to an out-of-band tone.

active component is a low-noise amplifier (LNA). The LNA is directly fed by the antenna signal through a via without any matching network choosing as antenna impedance the optimal impedance $(35.05-j 17.644) \Omega$ for minimum noise figure. No filtering is provided by intermediate stages in the DUT. This

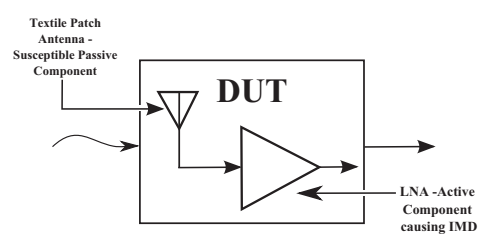

Fig. 5: Active antenna: passive textile antenna and integrated LNA.

DUT is now placed in the anechoic chamber set-up of Fig. 6. The multi-frequency signals are transmitted by a standard gain horn (SGH) letting them impinge in the far-field on the DUT. In our application, the DUT antenna has maximum gain in the forward direction with no other significant lobes present. Hence, the considered illumination will be the worst case scenario. The horn is connected to a cascade of two highly-linear power amplifiers and subsequently to an Agilent N5242A PNA-X network analyzer used as multi-frequency generator. The in-the-band frequency range is defined by its lower $f_{L}=2.4 \mathrm{GHz}$ and upper frequency $f_{H}=2.5 \mathrm{GHz}$, i.e. the (slightly extended) ISM-band.

\section{THREE SCENARIO COMPLIANCE TESTING OF THE ACTIVE ANTENNA}

\section{A. Equivalent Isotropically Radiated Power and Spurious Free Dynamic Range}

We now experimentally characterize the DUT of Fig. 5 in terms of the three scenarios put forward above. To do so, we need a sufficiently strong incident signal. To this end, two highly linear power amplifiers are cascaded (see Fig. 6): one

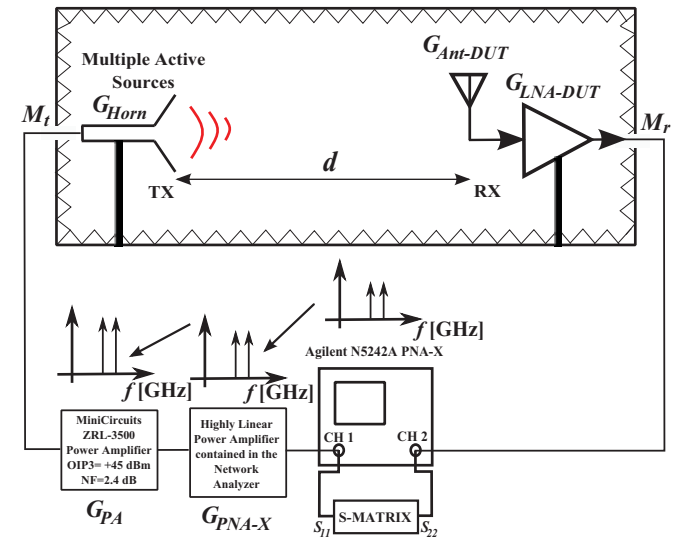

Fig. 6: Measurement set-up.

that is part of the Agilent N5242A PNA-X Network Analyzer and a Mini-Circuits (MC) ZRL-3500 with a maximum gain of $G_{P A}=21 \mathrm{~dB}$ and with a linearity far superior to that of the LNA that is part of our DUT. When using the MC amplifier in cascade with the PNA-X amplifier, careful experiments have revealed that the input power at the MC amplifier must be restricted to $0 \mathrm{dBm}$ to make sure that no relevant IMD turns up in the network analyzer-MC amplifier cascade and that the only IMD measured will indeed originate from the LNA in the DUT. To characterize the effect of out-of-band signals, we also need a suitable measure to decide whether the power level of the occuring unwanted signal is judged to be unacceptable. To this end, we use the Spurious Free Dynamic Range (SFDR) [6], here defined as the ratio between the desired component and the highest spurious component leaking into the band(s) of operation. By way of example, we require the SFDR to be higher than $40 \mathrm{~dB}$. To define the relevant power range of desired and unwanted signals incident from the SGH onto the DUT we rely on the Equivalent Isotropically Radiated Power (EIRP) [7], being the amount of power that the standard gain horn emits to produce the peak power density in the direction of maximum antenna gain. The EIRP is given by

$$
\begin{array}{r}
E I R P[\mathrm{dBm}]=P_{\mathrm{in}[P N A-X]}[\mathrm{dBm}]+G_{P A}[\mathrm{~dB}] \\
+G_{H O R N}[\mathrm{dBi}]-L_{[\text {cables }]}[\mathrm{dB}]-M_{t}[\mathrm{~dB}],
\end{array}
$$

with $G_{H O R N}$ the gain of the SGH (Scientific Atlanta SGH1.1 or 1.7 , depending on the desired excitation frequency), $G_{H O R N}=15.35 \mathrm{dBi} ; G_{P A}=21 \mathrm{~dB}$, the MC power amplifier gain; $L_{\text {cables }}=-0.06 \mathrm{~dB}$, the cable losses; with $M_{t}=$ $-0.1016 d B$, the transmission mismatch and with $P_{i n[P N A-X]}$ the input power generated by the PNA-X (ranging between 0 and $20 \mathrm{dBm}$ ). All numbers were determined at $2.45 \mathrm{GHz}$. The maximum allowed EIRP in the $2.45 \mathrm{GHz}$ ISM band is limited in Europe to $+20 \mathrm{dBm}$ by ETSI EN 300328 and by the US FCC Rules, Part 15.247, to $+36 \mathrm{dBm}$.

\section{B. Scenario 1}

As an example, $f_{0}=1.225 \mathrm{GHz}$ was used. While sweeping the input power, an in-the-band harmonic at $2 f_{0}=2.45 \mathrm{GHz}$ 


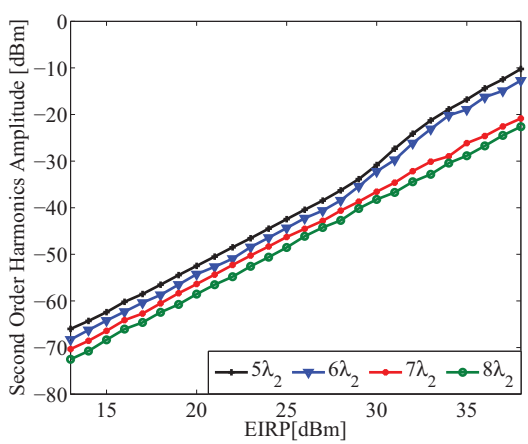

Fig. 7: Second harmonic's power versus distance between SGH and DUT.

is observed. The test was performed for different distances between the $S G H-1.1$ standard gain horn and the DUT. Fig. 7 shows the second-order harmonic's power over a range of distances between SGH and DUT from 8 down to $5 \lambda_{2}$ with $\lambda_{2}$ the free-space wavelength at $1.225 \mathrm{GHz}$. The power of the second-order harmonic remains smaller than $-52.47 \mathrm{dBm}$ for $d=5 \lambda_{2}$ and for an EIRP of $+20 \mathrm{dBm}$. To reach an SFDR of $40 \mathrm{~dB}$ i.e. our proposed threshold limit, the desired signal should reach at least $-12.47 \mathrm{dBm}$ for the DUT to pass the test.

\section{Scenario 2}

In this scenario, two (equal power) tones, radiated by the $\mathrm{SGH}$ are used. The frequencies of these tones are selected to be $f_{2}=2.395 \mathrm{GHz}$ and $f_{1}=2.405 \mathrm{GHz}$. The EIRP varies from $+10 \mathrm{dBm}$ up to $+37 \mathrm{dBm}$. The distance between the SGH-1.7 horn and DUT was varied from 9 to $12 \lambda_{1}$ (freespace wavelength at $2.45 \mathrm{GHz}$ ). Fig. 8 shows a sample result for a distance of $10 \lambda_{1}$ and EIRP levels of +27 and +30 $\mathrm{dBm}$. For an SFDR threshold of $40 \mathrm{~dB}$, and depending on the distance between the radiating element and the DUT, extensive measurements show that the SFDR stays above the threshold for EIRP values smaller than $32 \mathrm{dBm}$ and for a distance larger than $9 \lambda_{1}$.

\section{Scenario 3}

This last scenario concentrates on how desensitization affects the SFDR. The most critical configuration is found when a strong interfering signal (high EIRP) is present near the boundaries of the frequency band of operation, simultaneously causing IM products and desensitization in the DUT. More in particular, at higher EIRP-values, the power of the main tone $\left(f_{1}\right.$, see scenario 2$)$ no longer increases at the same rate as for the lower EIRP values while the intermodulation products grow faster. To characterize this phenomenon an important parameter describing the behavior of the DUT under the variation of EIRP is introduced: the Tone Power Variation $T P V_{n}$, defined as

$$
T P V_{n}=P_{\text {Tone }, n} / E I R P \mid e_{\text {constant path-loss, }}
$$
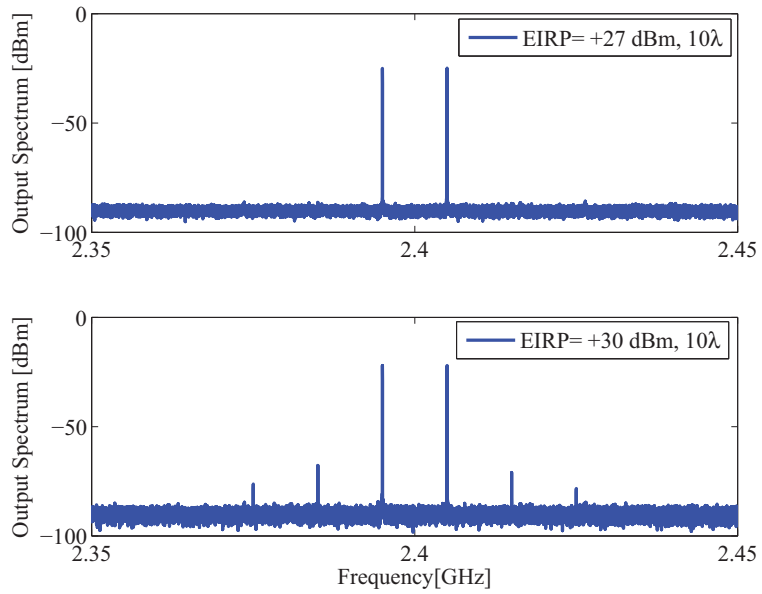

Fig. 8: Output spectrum for the excitation by two equal power tones in the proximity of the lower side of the frequency band with $\mathrm{EIRP}=+27$ $\mathrm{dBm}$ and $+30 \mathrm{dBm}$ respectively, using $d=10 \lambda_{1}$.

with, for our purposes, either the power $P$ of the main tone $(n=1)$ or of the third-order intermodulation product $n=3$. Fig. 9 shows $T P V_{1}$ and $T P V_{3}$ as a function of EIRP, for different distances between transmitter and DUT. In particular, by calculating this variation we can identify the power level at which the amplification starts to decrease for each transmitterDUT separation. When increasing the EIRP level we normally expect the power of the main tone to increase by the same quantity and the third-order intermodulation product to grow cubically until the LNA contained in DUT desensitizes due to saturation, exhibiting lower gain, resulting in a higher growth rate for the intermodulation products and in a lower growth for the main tones. This is clearly visible in Fig. 9: around +30 $\mathrm{dBm}$ of EIRP (for the closest distance between the test antenna and the DUT equal to $9 \lambda_{1}$ ) the LNA starts to desensitize.

\begin{tabular}{|c|c|c|c|c|}
\hline distance & $E I R P_{1}$ & $E I R P_{2}$ & $3^{\text {rd }}[\mathbf{d B m}]$ & $5^{\text {th }}[\mathbf{d B m}]$ \\
\hline $9 \lambda$ & +27 & +17 & -83.792 & NFL \\
\hline $9 \lambda$ & +30 & +20 & -60.39 & -70.27 \\
\hline $10 \lambda$ & +27 & +17 & -84.72 & NFL \\
\hline $10 \lambda$ & +30 & +20 & -76.92 & -79.52 \\
\hline $11 \lambda$ & +27 & +17 & -84.23 & NFL \\
\hline $11 \lambda$ & +30 & +20 & -79.238 & -82.381 \\
\hline $12 \lambda$ & +27 & +17 & -86.461 & NFL \\
\hline $12 \lambda$ & +30 & +20 & -82.652 & -85.331 \\
\hline
\end{tabular}

TABLE I: In-band amplitude of 3rd and 5th order interm. products.

This effect will be even more pronounced when considering a dual-tone co-located transmitter illuminating the DUT, as in the second scenario, but now assuming the out-of-band tone to be much higher in power than the desired signal. In particular, here we choose this unwanted component to be 10 $\mathrm{dB}$ higher in power. $E I R P_{1}$ represents the power level applied to the out-of-band tone, while $E I R P_{2}$ represents the power level applied to the in-the-band component. In the top part of Fig. 10 we observe that the intermodulation products occur at 

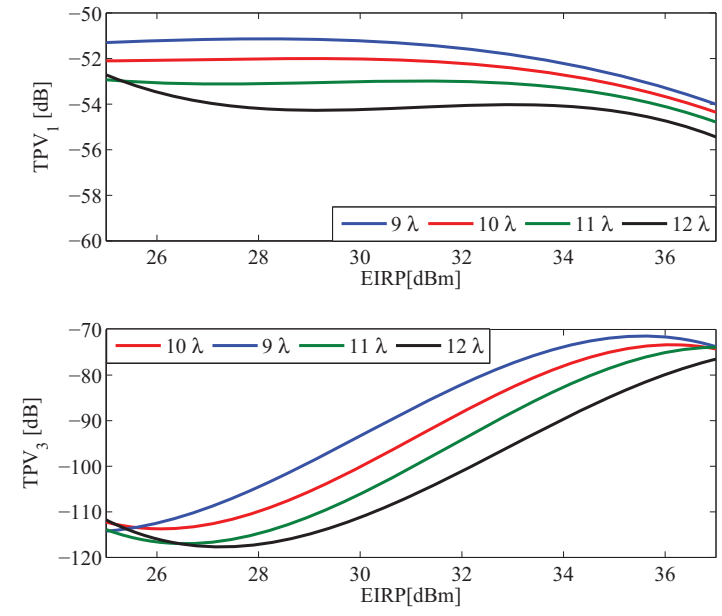

Fig. 9: $T P V_{1}$ and $T P V_{3}$ as a function of EIRP and for variable distances $\lambda=\lambda_{1}$ between horn and DUT.
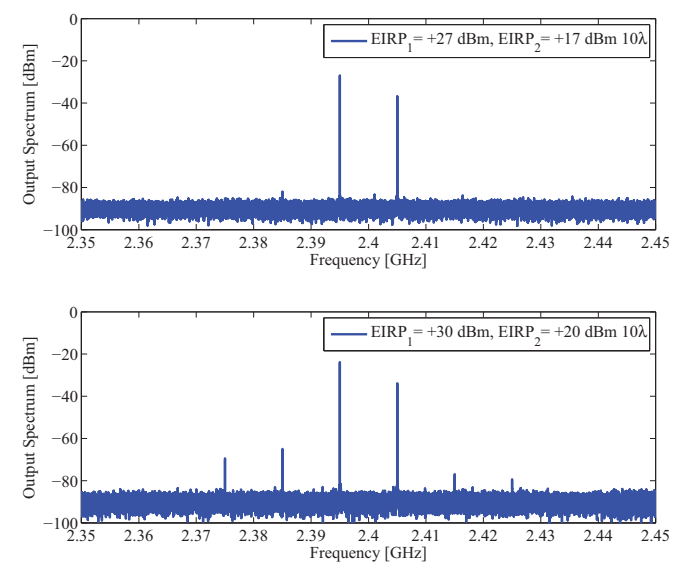

Fig. 10: Output spectrum for the excitation by two tones with different power in the proximity of the lower side of the frequency band, for $E I R P_{1}=27 \mathrm{dBm}, E I R P_{2}=17 \mathrm{dBm}$ and for $E I R P_{1}=30 \mathrm{dBm}$, $E I R P_{2}=20 \mathrm{dBm}$, respectively.

the same level as the noise floor (NFL), similar to the case with equal power for the out-of-band and in-the-band component. In Table I, some further data are given to illustrate the effect of distance. In Fig. 11 we notice that by increasing the $E I R P_{1}$ to resp. $+32 \mathrm{dBm}$ and $+34 \mathrm{dBm}$, and $E I R P_{2}$ to resp. $+22 \mathrm{dBm}$ and $+24 \mathrm{dBm}$, the in-the-band intermodulation product rises to $-62.25 \mathrm{dBm}$ and $-51.07 \mathrm{dBm}$, respectively. We observe that the third-order intermodulation product grows faster than cubically due to the influence of the out-of-band component. This phenomenon tends to be more noticeable when increasing the EIRP level. Indeed, for $E I R P_{1}=+32 \mathrm{dBm}$ we observe a difference of $2.39 \mathrm{~dB}$ between the expected in-the-band intermodulation product and the measured one. For the case $E I R P_{1}=+34 \mathrm{dBm}$ the difference equals $3.13 \mathrm{~dB}$. This effect quickly reduces the available SFDR. Indeed, for $E I R P_{1}=$ $+32 \mathrm{dBm}$, the SFDR level equals $30.85 \mathrm{~dB}$, whereas when going to $E I R P_{1}=+34 \mathrm{dBm}$, the SFDR drops to $18.98 \mathrm{~dB}$.
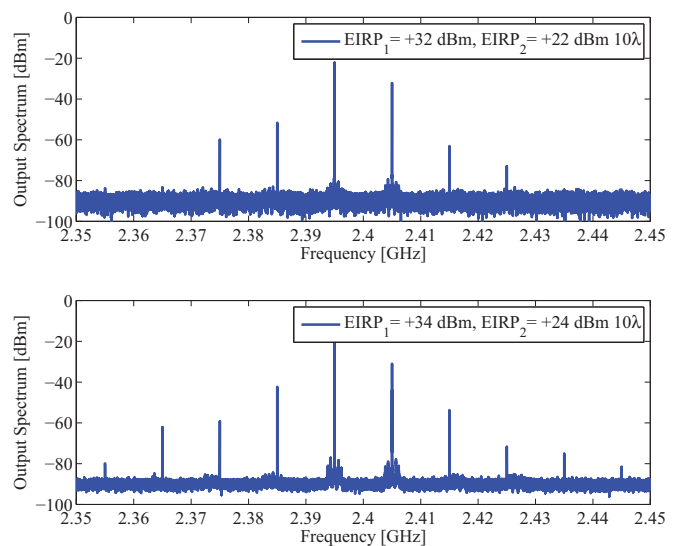

Fig. 11: Output spectrum for the excitation by two tones with different power in the proximity of the lower side of the frequency band and resp. for $E I R P_{1}=32 \mathrm{dBm}, E I R P_{2}=22 \mathrm{dBm}$ and for $E I R P_{1}=$ $34 \mathrm{dBm}, E I R P_{2}=24 \mathrm{dBm}$

\section{CONCLusion}

Few EMC test practices involve multi-source/multifrequency testing. We presented a new approach dealing with intermodulation distortion phenomena due to multiple colocated sources, suited for any DUT composed of at least one active non-linear component and a passive susceptible element, e.g. an antenna. An active textile antenna in the $2.45 \mathrm{GHz}$ ISMband was used as an illustrative example. Knowledge about leakage of undesired signals into the DUT's frequency band of operation can be obtained by using the SFDR as a suitable measure both for a single out-of-band or a combination of an in-the-band and an out-of-band frequency illumination. The influence of strong transmitters, desensitizing the LNA, on the relative importance of intermodulation products in the DUT's output spectrum was also examined using the Tone Power Variation parameter as a measure for the desensitization.

\section{REFERENCES}

[1] A. Rembovsky, A. Ashikhmin, V. Kozmin, S. Smolskiy, Radio Monitoring: Problems, Methods and Equipment, 1st ed. Springer, 2009.

[2] K. A. R. P. Cruz, N. B. Carvalho, "Designing and testing software designed radios," IEEE Microwave Magazine, pp. 83-94, June 2010.

[3] M. Mardiguian, "Combined effects of several simultaneous, EMI couplings," IEEE Int. Symp. on EMC, vol. 1, pp. 181-184, August 2000.

[4] A. Biondi, L. Valozzi, F. Declercq, H. Rogier, and D. D. Zutter, "EMC aware design and testing of intermodulation distortion under multiple colocated sources illumination," IET Science, Measurement and Technology, pp. 105-112, March 2012.

[5] F. Declercq and H. Rogier, "Active integrated wearable textile antenna with optimized noise characteristics," IEEE Trans. Antennas Propag., vol. 58, no. 3, pp. 3050-3054, Sep. 2010.

[6] D. M. Pozar, Microwave Engineering, 4th ed. New York: John Wiley \& Sons, 2012.

[7] C. A. Balanis, Antenna Theory: Analysis and Design, 2nd ed. New York: John Wiley \& Sons, 1997. 